\title{
Real-Time Rendering of Daylight Sky Scene for Virtual Environment
}

\author{
Changbo Wang \\ Software Engineering Institute of East China Normal University, Shanghai, China \\ cbwang@cad.zju.edu.cn
}

\begin{abstract}
Realistic rendering of sky scene is important in virtual environment. Traditional methods are mostly based on skybox, thus failing to realistically simulate the change of sky scene under different weathers and conditions. In this paper, a new sky light model under different weathers for virtual environment is proposed. We first analyze the atmosphere characters of sky scene, and calculate the light path and light intensity into eyes through the atmosphere considering atmospheric scattering and refraction. Then for different weathers, by adapting a path tracing algorithm, the intensity distribution of sky light is gained. we also adopt the scattered volume model and GPU techniques to accelerate the calculation. Finally, various sky scenes in sunny day, foggy day, raining day and that with rainbow under different conditions are realistically rendered in real time.
\end{abstract}

\section{Introduction}

Real-time rendering of outer-scene has always been a hotspot in virtual environment. Although the sky is one of the most common scene in virtual rendering systems, the sky scenes are quite difficult to be modeled and rendered realistically due to the complex mechanisms of interactions among the molecules, particles, light and the environment. A realistic sky scene will greatly improve the reality of the virtual environment when walkthrough.

Recently skybox technique is mainly used in virtual environment when rendering sky scene, but due to the simpleness of skybox, it could not show the realistic changes of sky scene in different weathers, such as sunny day, foggy and raining day, snowing day, et al. In order to simulate the dynamic changes of the sky scene, we must consider the physical character of light and particles in atmosphere, especially the scattering and refraction of sky light. For atmospheric scattering, in 1986, Max et al [1]introduced a single scattering model for light diffusion to generate haze in the atmosphere. in 1993, Nishita et al [2] proposed a metaball model to render the scattering effect of atmosphere which is based on the change of atmosphere density. Later, they introduced a method taking into account the multiple anisotropic scattering to render clouds and sky. In 2000, Dobashi et al [3] proposed an easy method for rendering shadows of clouds and shafts of light. Dobashi et al [4] proposed a fast rendering method for atmospheric scattering effects by using graphics hardware. Jackel et al. [5] simulated 
the Mie Scattering in 1997, and Nishita [6] used an improved sky illumination model to render the fog scene. But this approach of calculating the scattering effect for air particles is quite time-consuming. Nakamae et al.[7] proposed a continuous tone representation of the illuminated object by sky light. Sun et al. [8] proposed practical analytic method to render the single scattering in fog, mist and haze in real time. Nseda and Volksan [9] have calculated the atmosphere refraction and compared with experimental data, but they could not simulate it. By now little works have been reported about the real-time rendering of sky scene under different weather conditions.

In this paper, we propose a new physically based approach to model and render the sky scene under different weathers. We first analyze the character of atmosphere in sky scene, and establish a spacial sky light model considering atmospheric scattering and refraction. For different weathers, by adapting a path tracing algorithm, the intensity distribution of sky light into eyes is calculated. Finally, various sky scenes in sunny day, foggy day, raining day and that with rainbow under different conditions and different viewpoints are realistically rendered in real time.

The reminder of this paper is organized as follows: the next section we describe the sky light model considering the scattering and refraction. In section 3, we calculate the sky light and rendering different sky scene under different weathers. The implementation details are described in section 4. Experimental results with dynamic real-time rendering of sky scene are presented in section 5. Conclusions and discussion for future works are given at last.

\section{Modeling of Sky Scene}

\subsection{The Character of Atmosphere in Sky Scene}

The earth's atmosphere is categorized into 4 layers - troposphere, stratosphere, mesosphere and thermosphere. The earth's atmosphere is composed of many gases. Gravity holds the atmosphere close to the earth's surface and explains why the density of the atmosphere decreases with altitude. The density and pressure of the atmosphere vary with altitude and depends on solar heating and geomagnetic activity.

In addition to the various gases, atmosphere also contains water vapor, dust particles, etc. The molecules and particles absorb energy at discrete wavelengths, which are determined by their internal properties. In addition to absorption, molecules and particles also scatter energy out from its original direction. Suns white light is scattered into the viewing ray as shown in Figure 1. The scattered light is received at the earths surface from all directions as diffuse skylight or daylight.

On rainy environment, there are an enormous number of particles floating in the air. The density of raindrops is decreased exponentially as the increase of the raindrops' size. Although big raindrops account for only a small portion in rainfall, their effects on the scattering of lighting in the whole scene are apparent. 
We adopt the following statistical model for numerous raindrops in a rain[11]:

$$
F(r)=\left[1-e^{-\left(\frac{r}{a}\right)^{m}}\right]
$$

where $F(r)$ is the accumulated mass of raindrop with radius from zero to $r, r$ is the radius of a raindrop, $m$ is the exponent, for rain, $m$ is equal to 2.25, $a$ is the parameter about the amount of auriferous intensity, and its value depends on the rainfall amount. In this way, we are able to generate the spatial distributions of raindrops with different radii, and simulate different sizes of particles in the virtual environment.

\subsection{The Atmosphere Scattering and Refraction}

For a clear sky, various types of atmospheric particles are responsible for the scattering. There are mainly two kinds of scattering: Rayleigh scattering and Mie scattering.

Particles smaller than the wavelength and usually less than 0.1 times the wavelength of light exhibit Rayleigh scattering [3]. Rayleigh scattering is observed by molecules in the earth's atmosphere. The amount of scattering for such particles is inversely proportional to the 4-th power of the wavelength.

Larger particles scatter strongly in the forward direction and this scattering phenomenon is called Mie scattering. The scattering is inversely proportional to the second order of the size of the particles and is independent of wavelength. When foggy or raining day, there are more Mie scattering.

For different weathers, the particles is changed great, so we should extend the conventional single particle scattering model to accommodate the multiple particle scattering.

Above the light is taken as line when they travel through atmosphere. But in fact they are curve. Atmospheric refraction is responsible for the bending of light-rays in the atmosphere. It is a result of the continuous decrease in the refractive index of the air as a function of altitude. A well-known consequence of this phenomenon is the apparently elliptic shape of the setting or rising sun, and the color of sun is also more red when rising.

The refractive index of air of the Earth is very close to 1, and depends slightly on its pressure and temperature following Edlen's semi-empirical law [10], as shown in Eq. (2):

$$
n=1+10^{-6}\left(776.2+4.36 \times 10^{-8} \nu^{2}\right) \frac{P}{T}-0.1127 \frac{e}{T}
$$

In the above formula, $\nu$ is the wave-number of the light in $\mathrm{cm}^{-1} . P$ is the pressure in $k P a, e$ is vapor and $T$ the temperature in $K$. Since the pressure and temperature varies within the atmosphere, we get a refractive index gradient which is responsible for atmospheric refractions. Although the variations in $\mathrm{n}$ are quite small, the large distances travelled by light-rays in the atmosphere makes refraction effects observable and sometimes important.

We can derive the quantitative formula of refraction index in the term of air pressure, temperature, and vapor along with the attitude under different 
weather conditions, and then calculate the refractional track of light through the atmosphere to simulate the sky scene. In order to improve the calculate speed, we can divide the earth attitude into several layer. These layers have slightly different refractive indices.

\subsection{Calculation of Light Intensity}

Figure 1 shows the path of a single beam of sky light to the eyes of the viewer. All particles distributed along the sight ray will transmit the scattered light to the eye and these intensities are integrated by the following equation:

$$
\begin{gathered}
I_{v}=\int_{0}^{2 \pi} \int_{-\frac{\pi}{2}}^{\frac{\pi}{2}} \int_{s_{c p}=s}^{s_{v}} I_{\alpha}\left(S_{c p}\right) \sum_{i=0}^{N_{s}-1}\left(D_{\alpha, i}[\lambda, \theta] * p_{i}\left(S_{c p}\right)\right) \\
* \exp \left[-\sum_{i=0}^{N_{s}-1} \gamma_{i} \int_{s_{c p}}^{s_{p}} p_{i}\left(S_{b}\right) d S_{b}\right] d S_{c p} d \alpha d \delta
\end{gathered}
$$

where $D_{\alpha, i}[\lambda, \theta]$ is the phasic function of direction $\alpha, p_{i}\left(s_{c p}\right)$ is the density of particle $i$ at the position $s_{c p}, \lambda_{i}$ is the scattered coefficient of particle $i$, and $N_{s}$ is the number of scattered particles in the sky. The first term of the interior integral shows the summed intensity of scattered light by all particles located within the interval of the sight ray from $S_{c p}$ to $S_{v}$ with respect to the sky light in direction $\alpha$ interacting with the second term of the interior integral shows the energy attenuation from $s_{c p}$ to to $S_{v}$. Exterior integral account for skylights from all direction in the sky. When $N_{s}=1$, Eq.(3) is reduced to the single particle scattering model.

In Eq.(3), $I_{\alpha}\left(s_{c p}\right)$ is the intensity of the directional skylight. For different weathers, it can take different values.

Then we can calculate the light scattering through the whole ray path. Here we adopt the skylight model proposed by Nishita [1]. The sky is divided into several

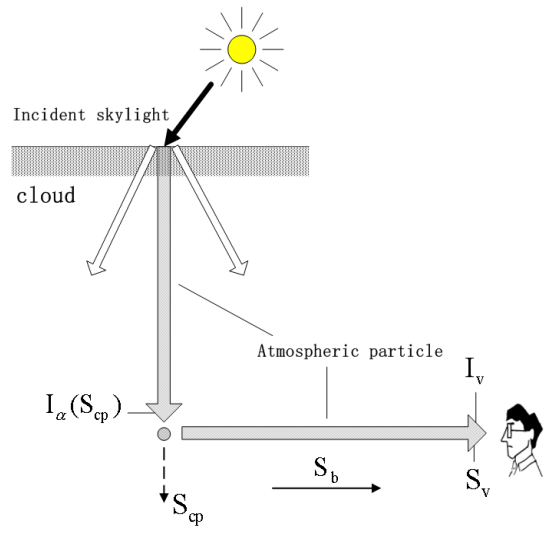

Fig. 1. The path of a single beam from the sun to the viewer's eye 
light straps, the intensity of each light strap is determined by the intensity of a sampling ray. Because the calculation of skylight is time-consuming, we simply assume the magnitude of the intensity of skylight at an arbitrary point as a function of height. Therefore, we pre-calculate the intensity at every height, then establish a lookup table.

Nevertheless, Eq.(3) does not present a direct solution and may cost much computation. it is then necessary to simplify this formula. Considering the diffuse illumination of the sky scene, we can ignore the direction of the skylights and regard the skylight uniformly from various directions as a diffuse light source and apply the sky luminance model directly. Then we can get the entire incident intensity of skylight at an arbitrary point to account for sky rays from different directions, and adopt a uniform phase function.

To avoid calculating the scattered light at each particle, we employ the concept of scattered volume [5]. Because the density of each kind of particles varies slowly. We piecewise sample the atmosphere by concentration of particles in the visual direction with respect to the distribution of the sky luminance. For each segment, we adopt the average value of the same kind of particles. The number of segments can be adjusted according to the precision. Finally, we can get the total intensity received by the viewer's eye.

\section{Rendering of Sky Scene}

\subsection{Rendering Algorithm of Path Tracing}

Since it is in outer-door and the atmosphere space is so big, the traditional rendering methods such as ray tracing and radiosity methods are not fit for. Here we propose a novel rendering algorithm: path tracing algorithm consider refraction.

The main idea of this algorithm is: firstly one ray is shot out from viewpoint to one sample point in the rendering screen plane, then conversely tracing this ray, which is along the refraction path $L(O P)$. For each point $S$ in the path, we can gain the refraction path $L\left(S P_{s}\right)$ from sunlight. Finally by the method in section 3.2 , calculate the scattering intensity in the whole path, that is integral along this path. The algorithm is shown in Figure 2. Further, we can use adaptive sample algorithm, that is more subtle sample in near the viewport, and coarse sample at the screen plane for these blue sky areas.

\subsection{Rendering Sky Scene Under Sunny and Foggy Day}

When calculating the light intensity, there is mainly Rayleigh scattering for sunny day, and more Mie-scattering for foggy sky.

For simplification, we use a uniform phasic function of direction, $D(g, \theta)$, to show both Rayleigh scattering and Mie scattering, which is used in Eq.(3) [5]. That is:

$$
D(g, \theta)=\frac{\left(3\left(1-g^{2}\right)\right.}{\left(2\left(2+g^{2}\right)\right)} \frac{\left(1+\cos ^{2}(\theta)\right.}{\left(1+g^{2}-2 g \cos (\theta)\right)^{3 / 2}}
$$




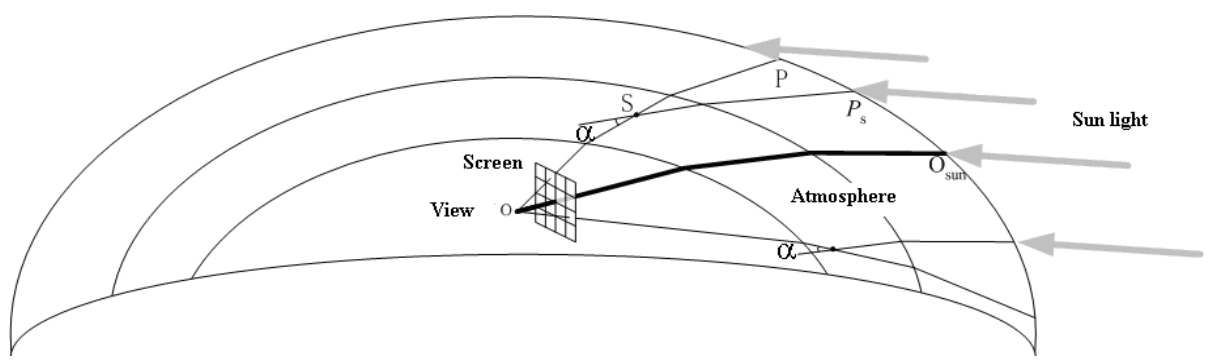

Fig. 2. Rendering algorithm of path tracing

Where $g=\frac{5}{9} u-\left(\frac{4}{3}-\frac{25}{81} u^{2}\right) x^{-1 / 3}+x^{1 / 3}, x=\frac{5}{9} u+\frac{125}{729} u^{3}+\left(\frac{64}{27}-\frac{325}{243} u^{2}+\frac{1250}{218} u^{4}\right)^{1 / 2}$. When $g=0$, the above equation is the Rayleigh scattering, and $u$ depends on the weather conditions, changing among [0.7, 0.85].

\subsection{Rendering Sky Scene Under Raining Day}

Unlike clear-days, there is normally no-direct solar light in raining scenes. Previous works mainly focus on calculation of light scattering by small particles in clear days, but the scattering mechanism in raining days is more complex due to the existence of different kinds of particles, such as raindrops, mists, aerosols, etc.

During raining days, the sky illumination is caused mainly by diffuse sky light rather than the direct solar light. All particles should interact with the sky light coming from different directions. Considering the diffuse property of the rainy sky, we can regard the skylight as a uniform light casting in from different directions within semi-sphere. Then we can express the entire incident intensity of skylight from different directions at an arbitrary point with a uniform phase function.

Just as in Eq.(3), for rainy day, the intensity $I_{v}$ should be attenuated through the path $S_{c p}$ to $S_{v}$, before the light is received by the viewer. This attenuation includes the scattering of small atmosphere particles and the refraction attenuation caused by encountering raindrops. The final intensity reaching the viewer's eye is:

$$
I_{v}^{\prime}=I_{v} R^{N(S V)}
$$

Where $N(S V)$ is the number of segments along the path. Here $R^{N(S V)}$ is the attenuation of raindrops, $R$ is the average refraction index of raindrops, $N(S V)$ is the number of rain streaks through the path $S_{c p}$ to $S_{v}$.

\subsection{Rendering Rainbow in Sky Scene}

Rainbow is a beautiful phenomenon that happens immediately after raining in sky scene. But it is not easy to realistically render in real time.

To render the rainbow, here we adopt the formula of light scattering discussed previously. However, in this case the incident light now is the parallel sunbeam 
and the scattered intensity is related to scattered angle. Also, we should consider the relationship between the amount of rainfall and the color width of rainbow. The distribution of the size of particles is determined by the distributing function of drops [10]. So Eq.(6)can be expressed as:

$$
I_{s}^{j}=I_{\alpha}^{j} \frac{1-\exp \left[-\left(s_{j+1}-s_{j}\right) \sum_{i=0}^{N_{s}-1} p_{i, j} \lambda_{i}\right]}{\sum_{i=0}^{N_{s}-1} p_{i, j} \lambda_{i}} \times \sum_{i=0}^{N_{s}-1} p_{i, j} D_{i}[\lambda, \theta]
$$

where $I_{\alpha}^{j}$ is the intensity of sunbeam, $\theta$ is the phasic angle from at which the sunbeam interact with the particles to transmit the color rays to the viewer's eye. We can produce the different effects of rainbow with respect to different amount of rainfall by adjusting the parameter $p_{i, j}\left(s_{v}\right)$.

As the scattering property is related to the size of a particles, we need to sample the atmosphere subtly, which would cost a lot of time. To balance our computing source and the final effect, we perform a fine sampling from $129^{\circ}$ to $138^{\circ}$ (scattered angle). This method not only accelerates the speed of rendering, but also seamlessly blends rainbow and the sky together well. At the same time, we can simulate the dynamic process of rainbow from appearing to vanish by adjusting the density of particles in the atmosphere.

\section{Implementation}

\subsection{Implementation Steps}

The implementation of our algorithm can be described as follows: (1) According to the weather type of sky scene, we set up the certain sky light propagation model. (2) For each sample point, we use the method discussed in Section 3.1 to calculate the trajectory and the light intensity of the light ray that passes through both the view point and that point, considering the refraction and scattering. (3) After rendering the scenes of the sea, landscape or building, and synthesizing them with the sky scene, we also render the raining and snowing based on particle systems, then we get the whole realistic dynamic sky scene under different weathers in virtual environment. (4) By adjusting the sky light model and the related parameters, we can simulate various dynamic processes of sky scenes.

\subsection{Luminance Transform}

By now, we have calculated the spectral intensity distribution of light incident into the eyes of an observer taking into account the interaction between light and various kinds of particles in virtual environment. But if we want to render the scattering effect realistically, we should transform the energy of spectrum to luminance perceived by the eyes.

According to the theory of human vision system(HVS), when perceiving an image, human eyes are more sensitive to the regions of moderate intensity than those of higher or lower intensity. Conventional linear transform method between 
the light energy and illumination is less adequate. Here we propose a new HVS based transform model as follows, which is in some extent like the form of bilogarithmic curve, that is:

$$
\left\{\begin{array}{l}
255 *\left(\log _{10}(x-a)+b\right)(x \geq a+1) \\
255 *\left(-\log _{10}(-x+a+2)+b\right)(x<a+1)
\end{array}\right.
$$

where $\mathrm{x}$ is the intensity, $\mathrm{a} \mathrm{b}$ is the curvilinear transformative parameters, which satisfies: $10^{b}=a+2 ; 10^{1-b}=I_{\max }-a\left(I_{\max }\right.$ is the maximum intensity $)$.

In this way, the effect of light scattering in virtual environments can be interpreted more realistically conformed to the optical characteristic of human eye.

\subsection{Other Acceleration Techniques}

In the before, we have used the scattered volume model and the path tracing algorithm to accelerate the calculation.

We also utilize GPU techniques to accelerate our scene rendering by storing the information of light etc. as texture in graphics card, and update it every frame. This greatly improve the rendering speed. The highlight on the car, rain splash effect on road and the other special effects in virtual environment are also generated with GPU acceleration.

\section{Results}

Based on the above models, we achieve the real-time rendering of sky scene on a PC with PIV3.0GHZ, 2.0GB, NVIDIA GeForce FX7900 graphics card, the average rendering speed reaches 20 frames per second.

Figure 3 shows the sky scene under sunny day. From it, we can see that the sky scenes are different between sea scene and landscape scene, that is because the vapors different, so atmosphere refraction is different and sky scene show different color. Figure 4 shows foggy and raining sky scenes with light scattering.
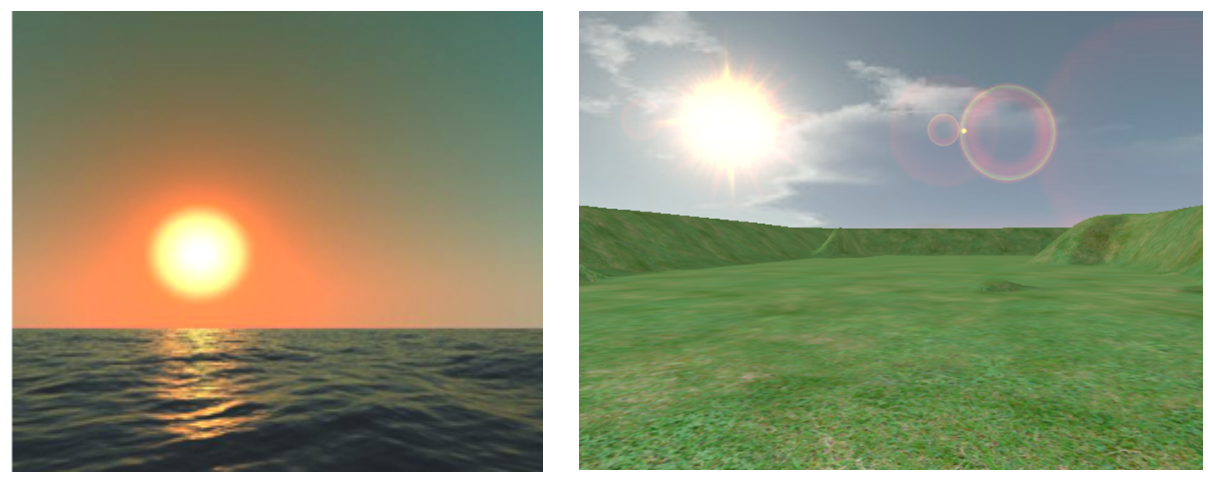

Fig. 3. Simulated sky scenes at sunny day 

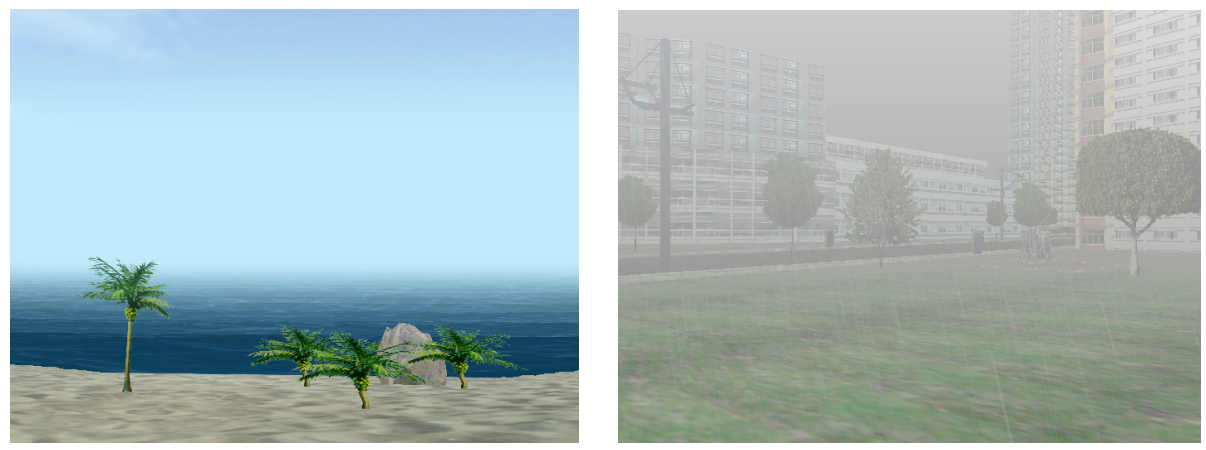

Fig. 4. Simulated sky scene under foggy and raining day
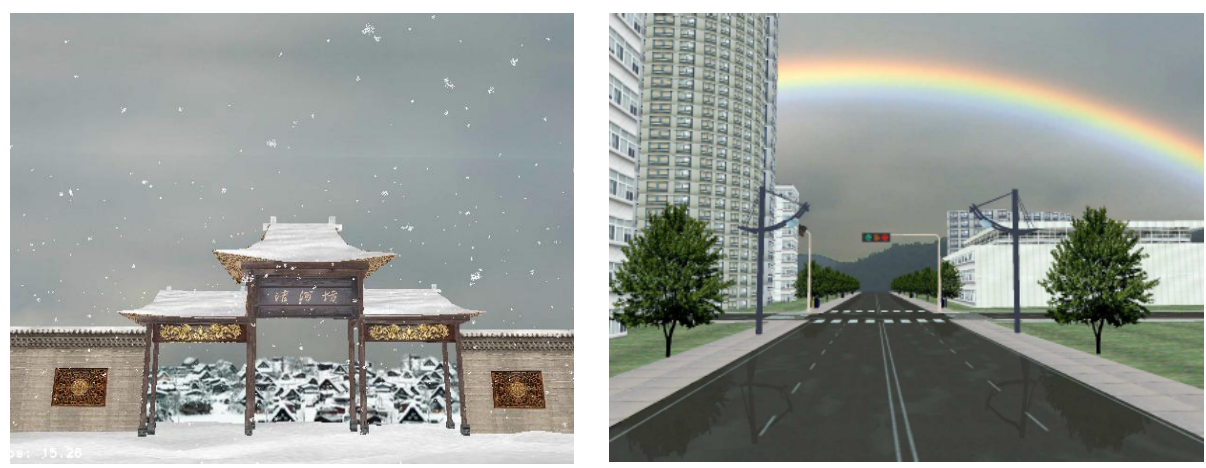

Fig. 5. Simulated snowing sky scene and the sky scenes with rainbow

The effect of light scattering is much better than that by directly applying the fog function of OpenGL. Figure 5 shows the snowing sky scene and the sky scene with rainbow after raining, we also can simulate the appear and disappear of rainbow. From these results, we can get that our simulated results is realistic.

\section{Conclusions}

we propose a new method to render the sky scene under different weather conditions for virtual environment. Firstly we consider the atmosphere scattering and refraction, build the sky light calculation model of sky scene. Then we use several methods to accelerate the rendering speed, including using the scattered volume model to simplify the calculation of scattering light intensity, and adapting a path tracing algorithm considering refraction to calculate the intensity distribution of sky light. Finally, various sky scenes in sunny day, foggy day, rainy day, and that with rainbow under different conditions and different viewpoints are realistically rendered in real time. 
Future works include: real-time rendering of cloud to improve the reality of sky scene; simulating the scene under different weathers and further accelerating the rendering speed by exploiting the power of the techniques of GPU.

\section{Acknowledgements}

This research was supported in part by 973 Program of China under Grant No. 2002CB312101, Natural Science Foundation of China under Grant No. 60603076.

\section{References}

1. Max, N.: Atmospheric Illumination and Shadows. Computer Graphics, 117-124 (1986)

2. Nishita, T., Takao, S., Tadamura, K., et al.: Display of the earth taking into account atmospheric scattering. In: Computer Graphics(ACM SIGRAPH'93), vol. 27(4), pp. $175-182(1993)$

3. Dobashi, Y., Kaneda, K., Yamashita, H., Okita, T., Nishita, T.: A simple, efficient method for realistic animation of clouds, pp. 19-28 (2000)

4. Dobashi, Y., Yamamoto, T., Nishita, T.: Interactive rendering method for displaying shafts of light, pp. 31-37 (2000)

5. Jackel, D., Walter, B.: Modeling and rendering of the atmosphere using miescattering. Computer Graphics Forum 16(4), 201-210 (1997)

6. Nishita, T., Dobashi, Y., Nakamae, E.: Display of clouds taking into account multiple anisotropic scattering and sky light, pp. 379-386 (1996)

7. Nishita, T., Nakamae, E.: Continuous tone representation of three- dimensional objects illuminated by sky light. Computer Graphics 20(3), 125-132 (1986)

8. Sun, B., Ramamoorthi, R., Narasimhan, S., Nayar, K.: A practical analytic single scattering model for real time rendering. Computer Graphics 24(3), 1040-1049 (2005)

9. Neda, Z., Volkan, S.: Flatness of the setting Sun. Journal of Physics 1, 379-385 (2002)

10. U.S. Standard Atmosphere 1976, U.S. Government Printing Office, Washington D.C. (1976)

11. Best, A.: The size distribution of raindrops. Quarterly Journal of the RoyaMeteorological 76(16), 16C36 (1950) 